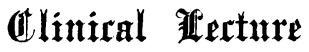 \\ on \\ THE RIGHT SIDE OF THE HEART AND ITS RELATION TO OVERSTRAIN.} $\mathrm{BY}$

WILLIAM RUSSELL, M.D., F.R.C.P.ED., PROFESSOR OF CIINICAL MEDICINE IN THE ONIVERSITY OF EDINBURGH, AND PHYSICIAN TO THE ROYAL INFIRMAIY,

I ASK your attention to-day to the consideration of the clinical phenomena which, when correctly interpreted, enable us to arrive at certain conclusions as to the condition of the right side of the heart. At the outset we must recall the anatomical relations of the right heart. A frontal view of the heart is made up of right auricle and ventricle, and, at its left limit, of a strip of left ventricle about half an inch in breadth. The origin of the pulmonary artery, from that part of the right rentricle called the conus arteriosus, is in the second left intercostal space; while its position, when not thus situated, can often be precisely determined by palpation and auscultation, as these define the point at which the closure of the pulmonary cusps is best felt and heard. The right ventricle lies under the third, fourth, and fifth spaces, extending in the fifth space to within half an inch to an inch of the apex of the left ventricle. The right border of the heart forms an arc the convexity of which is to the right, and which lies in the third, fourth, and fifth intercostal spaces, extending in the fourth to an inch or one and a half inches from the right sterual border.

\section{Methods of Examinaticn.}

\section{Percussion.}

The position of the left and right borders of the lieart can be determined by percussion in the different intercostal spaces. The right border is the more difficult of the two to determine, but I have explained to you on previous occasions how it can be done, and I have warned you that a right border represented as a line along the sternum may be the border of the right lung, but cannot be the right border of the lieart. The way you must look at this matter is, that the right border even of the normal heart is an inch or more beyond the sternum, and that you have to learn to find it. It is often considerably to the right of its normal limit.

\section{Inspection.}

Pulsation in the second left space is usually not due to the pulmonary artery, but to the conus arteriosus, and results from the fact that this part of the right ventricle dilates early, and that as it dilates it occupies the space carrying the origin of the pulmonary artery outwards and upwards, so that it may lie under the second rib, and be an inch or more to the left of the sternal border. Pulsation in the third, fourth, and fifth spaces, in the latter of these to within an inch of the apex, is always caused by the right ventricle, and all ideas based upen the phrase "diffuse apex beat" are erroneous. Such pulsation is a diffuse cardiac impulse, not a diffuse apex beat.

\section{Auscultation.}

In auscultating the heart the site of the apex of the left ventricle is auscultated, first, to determine the intensity of the first sound as produced by systole of the left ventricle; and, secondly, as the first step in determining whether a mitral lesion is or is not present. Having begun with the apex, and passing from it towards the sternum in the tifth, fourth, and third spaces, the loudness, faintness, or absence of the first sound is noted as an indication of the strength of the right ventricle. The old idea that the first sound as heard over the right ventricle was attributable to the left, as the thin-walled right ventricle was too wealk to prodnce sound, has been more or less abandoned. The right ventricle is now credited with at least some part in the production of the first sound as heard over it. I, however, ask you to go further than that. I would advise you to look at this matter from a different standpointnamely, that the first sound originating in the left ventricle and murmurs produced at the mitral orifice, as a rule and in the majority of cases, are not passed through the interventricnlar septum to the anterior wall of the right ventricle. 'The first sound as heard over the right ventricle is therefore not produced by the contraction of the left ventricle. As regards murmurs produced at the mitral orifice, I again impress upon you that, as a rule, these murmurs are not heara to the right of where the ventricular septum forms part of the anterior aspect of the heart. We have frequently in the wards cases-and there are two or more such cases there now-in which loud systolic mitral murmurs are not heard over the body of the right ventricle, while they are well heard outwards and upwards to the left of the apex. Such cases must be accepted as the standard by which we are to judge of the true area of limitation as well as the direction of propagation of mitral murmurs. You must abandon any erroneous views you may have learnt that mitral murmurs are commonly conducted over the whole precordia-conducted even to the base. The rule is that when a systolic mitral murmur is audible at the apex, and a murmur of the same time is heard to the right of the ventricular septum, this latter is a separate and superadded murmur, and is tricuspid in origin. In cases of marked incompetence the tricuspid murmur is often heard so high on the sternum that it may be thought to be aortic in origin. When the murmur thus raises the question of its seat of production, it will be found that on maliing the patient sit up in bed the murmur will disappear from the manubrium sterni if it is tricuspid in origin, and will reappear when the patient lies down. An aortic systolic murmur does not disappear and reappear with these changes in posture. The presenee of a tricuspid murmur can thus, in the majority of cases, be readily determined. In judging of the right heart the power to determine with accuracy the presence or absence of this murmur is essential, as it gives important evidence as to the condition of the right heart.

Let me impress upon you the points I have endeavoured to expound to you. They are: (1) That pulsation in the second, third, fourth, and fifth spaces, half an inch or more within a vertical line drawn through the apex, is caused by the right ventricle; and (2) that you have to differentiate tricuspid from mitral murmur on the lines I have formulated for you.

Abnormal Phenomena.

Visible Pulsation of Right Ventricle.

From these elementary points, which it has been necessary to dwell upon owing to the prevalence of misconceptions, we pass to the consideration of their bearing on the investigation of the condition of the heart. In the dorsal decubitus and in normal conditions the pulsation of the right ventricle is not visible save in persons with exceptionally small and thin chests. Visible pulsation means either that the right ventricle is dilated or that the left heart is dilated and has lifted the right ventricle into closer contact with the chest wall. With regard to the first of these explanations, I would warn you against a too facile belief in dilatation of the right ventricle; as a matter of fact, although various interesting changes occur in the right ventricle, dilatation such as is common in the left ventricle does not occur, for the simple reason that a very strong muscular band passes from wall to wall pre. venting it. The conus arteriosus readily dilates, and when it does so it carries the origin of the pulmonary artery outwards and upwards. With regard to the second explanation, it has this important practical significance that visible pulsation of the right ventricle may be proof of dilatation of the left heart, and herein, peihaps, lies its chief value as a clinical sign.

Accentuation of the Pulmonary Second Sound.

Returning to the auscultation of the right heart, you only require to be reminded that the second sound produced by the closure of the pulmonary cusps may be accentuated in its own area. The accentuation means heightened pressure in the pulmonary artery, and, when primary lung changes are excluded, it means increased fullness, with the consequent degree of increased difficulty, in the pulmonary circuit. Accentuated pulmonary second sound thus indicates the measure of impairment of the left ventricle which has determined the increased difficulty in the pulmonary circuit. Whenever the lelt ventricle is overstrained some degree of dilatation occn's. This necessarily means an increase of residua! blood in the ventricle, a corresponding measure of greater distensiou of 
the left auricle, and the increased engorgement of the pulmonary vessels which leads to the accentuated pulmonary second sound. You have here, then, a second means provided by the right heart of helping your judgement as to the state of the left lieart.

\section{Systolic Murmur in Pulmonary Artery.}

The presence of a systolic murmur in the second left intercostal space close to the sternum and strictly limited to this area is frequently noted. The conditions in which the murmur is present sinall be presently dealt with; meanwhile I ask you to note that the murmur is heard over the first part of the pulmonary artery; that this is the position of this part of the artery is confirmed not only by observations made after death, but also by the fact that, either by palpation or auscultation, or by both, the vibrations caused by the closure of the pulmonary cusps or the localized intensity of the sound enables us to locate with absolute precision the exact point where the artery joins the conus arteriosus. An interesting and important cliaracteristic of the murmur is that during inspiration, especially during a somewhat deep inspiration, the murmur disappears to reappear during expiration.

As to the conditions in which this murmur is present, chlorosis may be taken as an example. In chlorosis three murmurs may be present over the precordia-namely, a mitral systolic, a tricuspid systolic, and a pulmonary systolic. "The mode of differentiating tricuspid from mitral and from aortic svstolic nurmur has been already dealt with. That these murmurs are not due primarily to the im. poverishment of the corpuscles in haemoglobin is proved by the fact that the murmurs are pronounced in a case of chlorosis if the patient, usually a young woman, has been struggling to carry on her ordinary working duties in spite of an increasing sense of physical weakness. When such a patient comes into hospital and is kept in bed, two of the three murmurs commonly disappear in a few days, long before any material improvement has been effected in the blood. 'The mitral and tricuspid murmurs are due to dilatation and to such myocardial weakness as allows of leakage at both these orifices during systole. In other conditions of debility these murmurs may also be present. Apart, however, from the presence of mitral and tricuspid murmur, the systolic murmur in the pulmonary artery is frequently present as the only murmur heard over the precordia, and it is this murmur I specially want to explain the significance of and to show you that its presence enables us to judge of the condition of the left heart. The explanation I offer to you of the mode of production of this murmur is as follows: We have already seen that the right ventricle is lifted by dilatation of the left heart into close contact with the chest wall, so that its pulsations may be visible in the third, fourth, and fifth left spaces; systole of the right ventricle, especially systole of that portion of it called the conus arteriosus, carries the origin of the pulmonary artery obliquely downwards and to the right; if you endeavour to simulate this action, you will find that the anterior and posterior surfaces of the artery at its origin are approximated, and that a relative narrowing is thereby produced. This narrowing is, to my mind, undoubtedly the mechanism by which the murmur is produced. It explains the disappearance of the murmur during inspiration, for with inspiration the heart has a freer forward movement and the kink is prevented. This explanation gives an important value, and I believe the true value, to this murmur. Its presence becomes a proof of a dilated left heart with usually an enfeebled myocardium. It is present in anaemia, in febrile conditions, in debility, and as a result of heart strain. It belongs to the class of murmurs which are called functional to differentiate them from murmurs due to endocarditis and called organic. The murmur is present in cases of organic mitral lesion when the origin of the pulmonary artery has not been carried to the left and upwards by the dilatation of the conus arteriosus.

Pulmonary.Diastolic Murmur.

Pulmonary diastolic murmur not due to endocarditis at this oritice is, in my experience, very rare. I have, however, recently seen a case of the kind. The patient was a young Royal Field Artillery officer who had returned to light work after recovering from an extremely feeble heart following upon a febrile attack in Flanders. The light work had proved too much for him, and he showed a faint diastolic murmur over the pulmonary orifice, a faint systolic, tricuspid murmur, a right auricle considerably to the right of its normal position, and a marked ventricular systolic wave in the neck veins. This was a case of pure strain in a heart that had materially improved in myocardial strength but not sufficiently to withstand the strain of the work laid upon it. The murmurs disappeared under physical rest and medicinal treatment.

\section{Explanations.}

The explanations already given of the phenomena with which we have dealt may be supplemented with advan. tage. In the first place, feebleness or absence of the first sound over the right ventricle means weakness of the myocardium of that chamber, and in stout people it commonly means fatty infiltration. Cases of this kind die suddenly from some sudden physical effort, death resulting from right heart failure, not left failure. As we have seen, accentuation of the pulmonary second sound, systolic pulmonary murmur, systolic tricuspid murmur, diastolic pulmonary murmur are the auscultatory phenomena, having origin on the right side of the heart, which may be present. They all indicate strain, varying in degree, of course-strain in the sense that the myocardium is being called upon, or has been called upon, to do more than it can do comfortably or satisfactorily. Such strain leads to dilatation, sometimes of the left heart, at other times of the æight, sometimes of both. Some of the phenomena produced on the right side of the heart become the index and guide to strain of the left heart; these are especially accentuation of the pulmonary second sound, pulmonary systolic murmur, and pulmonary diastolic murmul. 'The presence of tricuspid murmur shows, of course, that the right ventricle is dilated, or atonic and enfeebled, either from initial strain or from the strain due to heightening of the pulmonary blood pressure the consequence of initial strain of the left ventricle. The auscultatory phenomena result from relaxation and dilatation, and it is that fact which gives them their clinical significance and importance. Visible pulsation of the right ventricle has the significance already dwelt upon, and it is sometimes more valuable as an index of left-sided than of right-sided dilatation.

\section{Venous Pulsation.}

I now ask your attention to a further source of information-to another set of phenomena from which important deductions can be made and which may lead very promptly to accurate diagnosis. I mean pulsation in the veins of the neck. There are two methods of making observations on these: the first is by means of the unaided eye. Fullness and pulsations, the rhythm of pulsations as timed by cardiac systole, whether they are entirely synchronous with systole, or what their time relation to systole may be, can be seen and noted, and let me impress upon you that this field of observation ought to be diligently explored by you. The second method of investigation is by means of that beautiful clinical instrument invented by Sir James Mackenzie, and known as Mackenzie's polygraph. The latter method gives details which the eye cannot provide; at the same time I repeat that the unaided eye carries us a long way.

Pulsations in the veins of the neck are of course determined by the right side of the heart. This simple proposition is fundamental, and when it is fully grasped the second step is inevitable-namely, that such pulsations can only be caused by contraction of the ventricle or of the auricle. Pulsations are therefore systolic in time when ventricular in origin; all other pulsations are diastolic in time, and have their origin in the auricle. In the consideration of this aspect of our subject $I$ confine myself to cases which are at present under observation, and I shall show you on the screen tracings made by means of the electro-cardiograph and by means of Mackenzie's polygraph.

CASE I.-R., a woman aged 65, had a pulse-rate of 40 when I first saw her. The pulse-rate corresponded with the number of ventricular systoles. On inspection of the veins in the neck there was visible a wave synchronous with rentricular systole, and between each of these another wave, which was presumably auricular systolic 
in time, and it was so regular that there was little donbt that the case presented the phenomena of a 2 to 1 heartblock. I sliow you tlie polygraph tracing (Fig. 1) in which

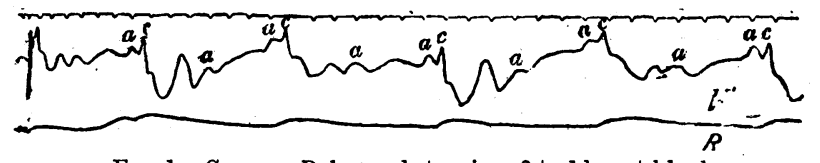

FIG. 1.-Case I. Polygraph tracing, 2 to 1 heart-block.

each wave marked $a$ is produced by the auricle which is contracting quite rhythmically; the wave $c$ is the com. municated wave produced in the carotid artery. A few
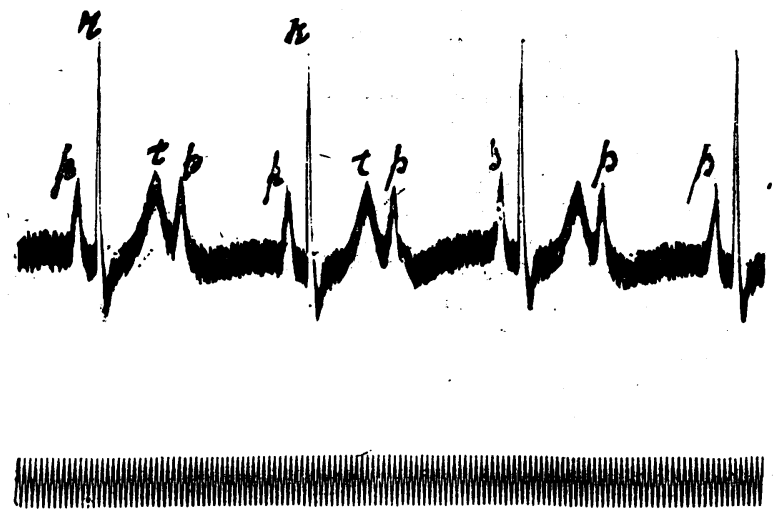

Fig. 2.-Case I. Electro-cardiogram, 2 to 1 heart.block.

days later the electro-cardiogram (Fig. 2) was taken, and you note that each wave $r$ which is near the commencement of the ventricular movement is preceded by two auricular contractions marked $p$. The diagnosis of a
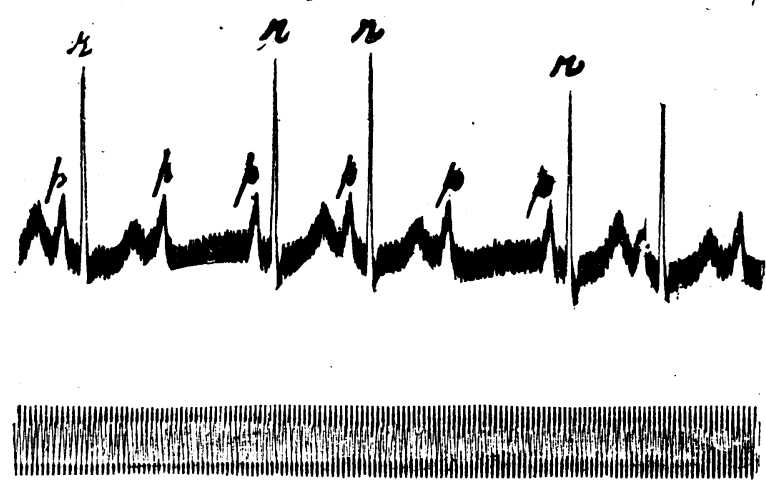

Fig. 3.-Case r. Alternate ventricular systoles preceded by two auricular systoles.

2 to 1 heart-block was thus confirmed. This patient's heart was improving, and even at the same séance as gave us Fig. 2, Fig. 3 was also obtained, and shows that only every alternate auricular systole $p$ was not followed by the ventricular wave $r$.

CASE II.-W., a man aged 60, with marked cardiac irregularity, showed in the neck to the unaided eye a confused succession of undulatory movements, in addition to the respiratory distensions and emptyings. The polygraph tracing from the neck and the radial artery (Fig. 4) gives the position of the communicated carotid pulsc

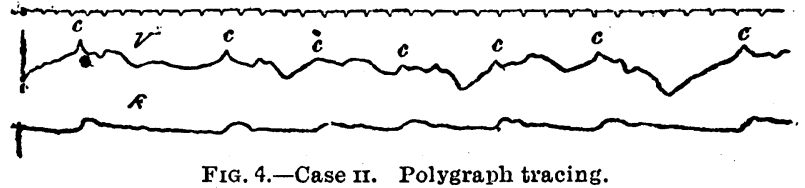

marked $c$, and you note there is an absence of the auricular wave present in the preceding tracings. In fact, there is no evidence here of rhythmic auricular systoles; there is practically what used to be called paralysis of the auricle, but is now known as auricular fibrillation: The electro-cardiogram (Fig. 5) bears out the diagnosis of extreme irregularity, the absence of a true
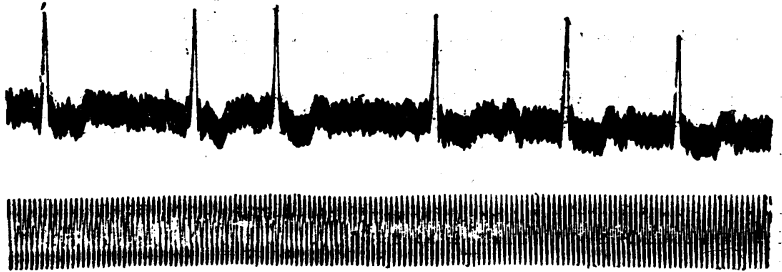

Fig. 5.-Case II. Electro-cardiogram, no auricle wave.

auricular wave, and the opinion that the patient had a fibrillating auricle.

CASE IIr. - S., a man aged 54 , with a systolic mitral murmur and a large heart, showed a ventricular systolic venous wave in the neck. The tracing (Fig. 6) shows the position of waves $a$ and $c$ as taken by the polygraph.

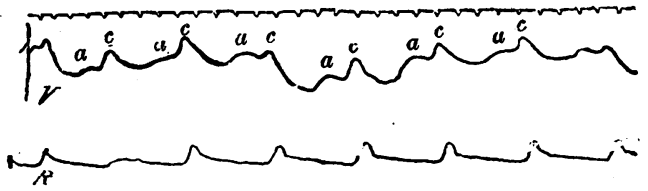

Fig. 6.-Case IrI. Polygraph tracing, in which the venous pulse seemed to be systolic.

The timing of visible venous pulsations is sometimes difficult, but in many cases ventricular systolic and auricular systolic waves can be separated, and their presence must be a measure of the fullness of the right side, especially of the right auricle.

I do not follow our subject further to-day, but you will understand that the study of the means by which the right side of the heart can be investigated, and the method of interpretation of the phenomena which I have pre. sented to you, are able to guide you to a true estimate of many a heart that will come under the consideration of some of you, probably in the near future. The matters I have dealt with lie at the foundation of a true understanding of overtaxed hearts.

\section{THE THEORY OF BLOOD PRESSURE MEASUREMENT}

With SPEctal Reference to the USE of Schemata aND Blood Pressure Instruments, TOGETHER WITH AX EXPLANation OF the Discordant Results arising FROM THE APPLICATION OF THESE INSTRUMENTS.

BY

LEONARD HILL, F.R.S., DIRECTOR, DEPARTMENT OF APPLIED PHYSIOLOGY, Y:D, EAL AND

JAMES M. McQUEEN, CaptaIN R.A.M.C.(T). RESEARCH FELLOW OF ABERDEEN ENIVERSITY.

THE study of blood pressure measurement has been of slow growth. The literature of the subject in past years shows how varying types of measuring instruments were employed, how the readings of systolic pressure obtained by them differed with the type of instrument; how with the same instrument discordant readings were obtained from arteries in different situations, where equality or something approaching to equality of reading might havo been expected.

Mucl attention has also been given to the fixing of the diastolic standard. Here also disagreements were to bo met with, disagreements both on the nature of the auditory and on the nature of the oscillatory index. Recent work, however, has made possible methods of accurately determining both the systolic and the diastolic pressure in the human subject.

Cousiderable progress, apart from the fixing of stanclards, has been made in the theory of blood pressure measure- 